\title{
Integrable microwave filter based on a photonic crystal delay line
}

\author{
Juan Sancho ${ }^{1, \star}$, Jerome Bourderionnet ${ }^{2, \star}$, Juan Lloret ${ }^{1}$, Sylvain Combrié ${ }^{2}$, Ivana Gasulla', Stephane Xavier², \\ Salvador Sales ${ }^{1}$, Pierre Colman², Gaelle Lehoucq ${ }^{2}$, Daniel Dolfi ${ }^{2}$, José Capmany ${ }^{1} \&$ Alfredo De Rossi ${ }^{2}$
}

The availability of a tunable delay line with a chip-size footprint is a crucial step towards the full implementation of integrated microwave photonic signal processors. Achieving a large and tunable group delay on a millimetre-sized chip is not trivial. Slow light concepts are an appropriate solution, if propagation losses are kept acceptable. Here we use a low-loss $1.5 \mathrm{~mm}$ long photonic crystal waveguide to demonstrate both notch and band-pass microwave filters that can be tuned over the $0-50-\mathrm{GHz}$ spectral band. The waveguide is capable of generating a controllable delay with limited signal attenuation (total insertion loss below $10 \mathrm{~dB}$ when the delay is below 70 ps) and degradation. Owing to the very small footprint of the delay line, a fully integrated device is feasible, also featuring more complex and elaborate filter functions.

\footnotetext{
${ }^{1}$ ITEAM Research Institute, Optical and Quantum Communications Group, Universidad Politécnica de Valencia, Camino de Vera s/n, 46022 Valencia, Spain. ${ }^{2}$ Thales Research and Technology, 1. av. A. Fresnel, 91767 Palaiseau, France. ${ }^{\star}$ These authors contributed equally to this work. Correspondence and requests for materials should be addressed to J.C (email: jcapmany@iteam.upv.es) or to S.C. (email: sylvain.combrie@thalesgroup.com).
} 
$\mathrm{M}$ icrowave photonics (MWPs) ${ }^{1-3}$, which brings together the worlds of radiofrequency engineering and optoelectronics, has attracted great interest from both the research community and the commercial sector over the past 30 years, and is set to have a bright future ${ }^{4}$. Its benefit stems from the fact that it enables the realization of key functionalities in microwave systems that are either complex or even not directly possible in the radio frequency (RF) domain. An important example is microwave signal filtering, which is ubiquitous in communications, radio-over-fibre, radar, radio astronomy and digital satellite communication systems ${ }^{5}$. Agility, reconfiguratibily and tunability are highly desirable features, which are, however, not straightforward to implement using traditional electronic circuitry, for example, microstrip ${ }^{6}$ or coplanar waveguide lines $^{7}$, substrate-integrated waveguides ${ }^{8}$ and superconducting circuits $^{9}$. To this end, approaches based on ferroelectric materials are being investigated, such as barium strontium titanate ${ }^{10}$, semiconductor varactors diodes ${ }^{6}$, metamaterials ${ }^{7}$, micro-electro-mechanical systems $^{8}$ and SiGe BiCMOS-compatible structures ${ }^{11}$.

However, only MWP approaches have provided, to date, promising results in terms of tunability, reconfigurability and broadband operation. MWP signal processors reported so far ${ }^{12-16}$ have relied on discrete optoelectronic devices and standard optical fibre delay lines, and components leading to bulky, expensive and powerconsuming architectures. Typical delay line footprints range from several tens of centimetres when using fibre Bragg gratings (FBGs) to a few kilometres when using standard optical fibres.

A solution to the above limitations of MWP filters is contingent on the possibility of incorporating this functionality into an application-specific photonic integrated circuit, which makes the former approaches based on extended delay lines inapplicable. Preliminary efforts towards integration have relied on resonant delay lines provided by ring cavities and coupled resonant optical waveguides ${ }^{17-20}$. These approaches suffer from an intrinsic trade-off between dispersion and bandwidth, preventing broadband operation. Thus, the cornerstone towards the integration of MWP filters resides in the availability of an integrated delay line, suitably designed to operate with MWP signals and providing enough dispersion. In this context, photonic crystal $(\mathrm{PhC})$ waveguides are potential candidates, as the dispersion profile can be tailored to an extent that is no match with other photonic structures 21,22 .

In this work, we provide the first ever demonstration of tunable and reconfigurable MWP filters based on a 1.5-mm-long GaInP/ GaAs highly dispersive $\mathrm{PhC}$ waveguide delay line optimized for RF signals. The reduced waveguide loss in the slow light regime, owing to both design and fabrication optimization, is crucial to achieve the large dispersion values required for the integrated delay line. The salient features include: notch and band-pass operation; a footprint reduced by several orders of magnitude compared to filters based on FBG or fibre delay lines; broadband operation up to $50 \mathrm{GHz}$; and tunable free spectral ranges over $70 \mathrm{GHz}$. In addition, there is clear potential for the improvement of the selectivity, the main to secondary side-lobe (MSSL) ratio and the Q factor, thereby leading to highperformance MWP filtering owing to compatibility with sources of optical frequency combs ${ }^{23}$.

\section{Results}

Basic concept. The most powerful and versatile approach for the implementation of MWP filters is that based on incoherent discrete signal processing ${ }^{24}$. In particular, finite impulse response ${ }^{5}$ filters combine at their output a finite set of delayed and weighted replicas or taps of the input optical intensity signal (a RF-modulated optical carrier $)^{5}$. The usual implementation of this concept in the context of MWPs can follow two approaches as shown in Fig. 1. In the first one (Fig. 1a), the delays set to multiples of $T$ are obtained, for instance, by means of a set of $N$ non-dispersive optical fibre coils where the length of the fibre in the $k$-th tap is $c(k-1) T / n$, where $c$ is the light velocity in the vacuum and $n$ is the refractive index. This simple scheme does not allow tuning, as this would require changing the value of T. An alternative approach (Fig. 1b) is based on the combination of a dispersive delay line and different optical carriers. Here the optical sources emit at different wavelengths so the spectral separation of a pair of consecutive modes implementing consecutive taps is $\Delta \lambda$, and the basic delay is given by $T=D L \Delta \lambda$, where $L$ represents the length of the dispersive element and $D$ its dispersion parameter. Thus, the value of the basic delay $T$ is changed by tuning the wavelength separation among the carriers, thereby allowing tunability ${ }^{25}$. Although in the first case the intensity or weight $a_{k}$ of the $k$-th tap can be changed by inserting loss/gain devices in the fibre coils, with the second approach, $a_{k}$ is readily adjusted by changing the optical power emitted by the optical sources $^{12,25}$. In this work, we focus on the approach of Fig. 1b, as it is more flexible.

Implementation of the $\mathrm{PhC}$ delay line. A PhC waveguide results from a missing line in a regular lattice of holes on a self-standing dielectric (here GaInP) slab, as shown in Fig. 2a,b. Owing to the inherently complex waveguiding mechanisms originating from Bragg scattering in the photonic lattice, the dispersion of such waveguides departs from that of their more conventional counterparts, such as, for instance, rib-loaded structures. A decade ago, it was demonstrated that in a $\mathrm{PhC}$ waveguide the group velocity might vary from $c / 5$ up to $c / 90$, thereby laying the foundations of a chipsized optical delay-line ${ }^{21}$. Since then, $\mathrm{PhC}$ waveguides have been intensively investigated for generating slow-light ${ }^{26}$.

Propagation loss in $\mathrm{PhC}$ waveguides can be as low as a few $\mathrm{dB} \mathrm{cm}^{-1}$ (ref. 27). However, it systematically increases when the group velocity is reduced, because of the enhanced impact of the fabrication imperfections, mainly roughness at the etched interfaces. This has been preventing the achievement of large optical delays with moderate attenuation and, thereby, practical use for implementing functions in the domain of optical signal processing until very recently ${ }^{28}$. By engineering the field distribution of the propagation mode, O'Faolain et al. ${ }^{29}$ have demonstrated a substantial reduction of backscattering and an improvement of the transmission of modes with group velocity smaller than $c / 30$ in silicon-based $\mathrm{PhCs}$.

It has been pointed out ${ }^{29}$ that the attenuation $(\mathrm{dB})$ per delay (ns) is more appropriate than attenuation per distance $\left(\mathrm{dB} \mathrm{cm}^{-1}\right)$, when referring to delay lines. In this respect, a remarkable figure of $25 \mathrm{~dB} \mathrm{~ns}^{-1}$ has been reported ${ }^{29}$. Still, here it is implicitly assumed that this figure, measured in short (a few hundreds of micrometres) waveguides, can be extrapolated to any length. Indeed, the theory supports this claim for single mode, missing line defect $\mathrm{PhC}$ waveguides, so long the disorder is assumed random and with the same statistics all long the waveguide ${ }^{30}$. It must also be pointed out that the attenuation (measured or calculated) is defined as an average over a large number. These points must be considered when considering the actual use of these waveguides in applications.

For application in MWPs, a tunable delay as large as $100 \mathrm{ps}$ is needed, which implies the use of a long waveguide, for example, in the millimetre range. Thus, the probability of multiple coherent scattering events is much stronger relative to a shorter (for example, $100 \mu \mathrm{m}$ ) waveguide that is operated at the same group velocity. This results in distortion of the transmission spectrum ${ }^{31,32}$, even leading to sharp resonant peaks, even when the group index $\left(n_{\mathrm{g}}=c / v_{\mathrm{g}}\right)$ is relatively small $(\sim 15)$. This is even more detrimental for applications than the mere (averaged) value of the attenuation.

Furthermore, the fabrication of long $\mathrm{PhC}$ waveguides implies a mechanical displacement of the sample; each time the maximum writing area of the electron beam system (typically a few hundreds of micrometres) is reached. That might lead to 'dislocations' (similarly to crystals) in the PhC pattern, thereby altering the statistics of the disorder along the waveguide. 


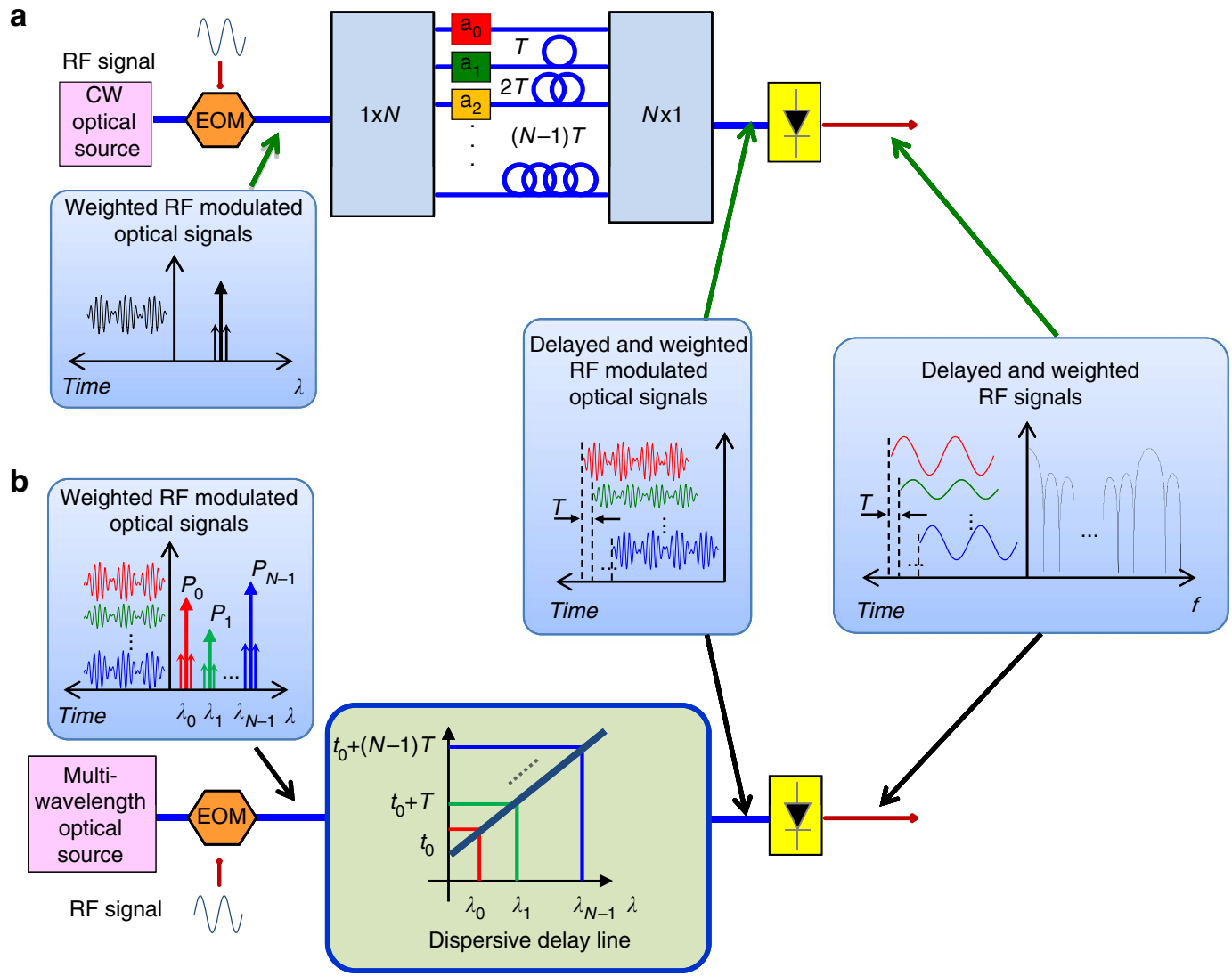

Figure 1 | General schematics of a MWP multi-tap filter. (a) Traditional approach based on a single optical source in combination with multiple nondispersive short fibre coils. The basic delay $T$ is implemented by means of a dispersionless fibre coil of length $c T / n$, where $c$ stands for the speed of light in vacuum and $n$ is the refractive index of the core. The RF-modulated optical signal is divided by a $1 \times N$ coupler into $N$ taps or samples. The weight of each sample can be changed by means of an individual attenuator or amplifier. For instance, the $k$-th tap is weighted in power by the attenuator/amplifier providing a value of $a_{k}$ and it is delayed by $(k-1) T$ by means of a fibre coil of length $(k-1) c T / h$. The signal samples are combined by means of a $N \times 1$ coupler and fed to the photodetector. The boxes connected with green arrows show the time and frequency domain structure of the signals at selected points of the layout. (b) A more compact approach based on a multi-wavelength optical source together with a single dispersive element. The optical sources emit at different wavelengths so the spectral separation of a pair of consecutive modes implementing consecutive taps is $\Delta \lambda$, and their basic delay is given by $T=D L \Delta \lambda$ where $L$ represents the length of the dispersive element and $D$ its dispersion parameter. Here, the intensity or weight $a_{k}$ of the $k$-th tap can be adjusted by changing the optical power emitted by the optical sources. The boxes connected with black arrows show the time and frequency domain structure of the signals at selected points of the layout.

For all these reasons, the suitability of a $\mathrm{PhC}$ waveguide as a delay line for MWPs must be assessed under more realistic conditions, as follows. First, the total insertion losses, including input and output coupling, must be considered relative to the actual time delay (for example, $100 \mathrm{ps}$ ), and not extrapolated. Second, the delay should be continuously tunable, for example, transmission should not change abruptly when changing the operating point of the device. Finally, the signal distortion, due to coherent multiple scattering, must be taken into account by characterizing the sample with a signal with realistic bandwidth.

Here, we report the design, fabrication and characterization of an optical delay line based on a $\mathrm{PhC}$ waveguide that meets these requirements. The use of the $\mathrm{PhC}$ waveguide was reported for the first time ${ }^{33}$ using a simple missing line defect $\mathrm{PhC}$ waveguide. The delay achieved there was a few tens of picoseconds, strongly distorted by Fabry-Perot interference due to reflections at the as-cleaved waveguide ends.

The waveguide design is characterized by an anti-symmetric shift of the first row of holes ${ }^{34-36}$, as indicated in Fig. 2c (see details in Methods). This modification of the waveguide symmetry induces a coupling between the even and odd mode resulting in a 'hump'-shaped dependence of the group index on the wavelength (see Fig. 2d). Details are discussed in Colman et al. ${ }^{36}$ The group delays for both TE (defined as the $E$ field parallel to the membrane and perpendicular to the direction of propagation) and TM modes have been measured with an interferometric technique (see Methods). The calculated dispersion for both TE and TM modes is also plotted, with excellent agreement.

The fabricated device is $1.5 \mathrm{~mm}$ long and contains mode adapters ${ }^{37}$, as shown in Fig. 2a, owing to which the total insertion loss (minimum value at low group index) is reduced to about $7 \mathrm{~dB}$ (from fibre to fibre, including propagation in the waveguide and fibre/ $\mathrm{PhC}$ coupling). In addition, undesirable Fabry-Perot fringes in the transmission spectra are removed, as apparent in Fig. 3a. The residual modulation results from multiple coherent scattering ${ }^{31}$. The corresponding group index is also plotted in the same figure. The important point here is the moderate $(3.5 \mathrm{~dB})$ optical transmission penalty, as the group velocity is reduced to $c / 14$. Thus, a delay of $70 \mathrm{ps}$ is achieved with total insertion loss $10 \mathrm{~dB}$ from fibre to fibre.

We point out that not only the improvement of the fabrication process is important but also the choice of the waveguide design has a role. Figure $3 \mathrm{~b}$ represents the transmission plotted against the 


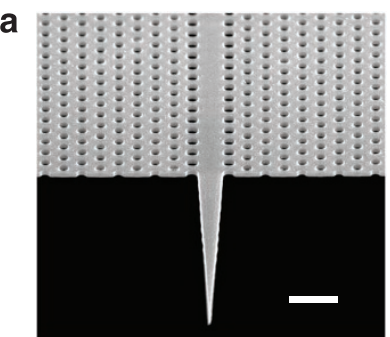

C

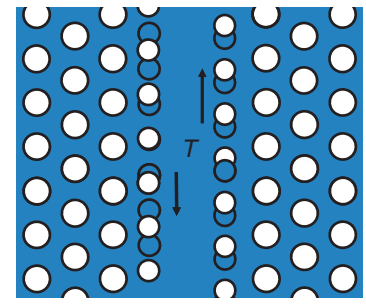

b

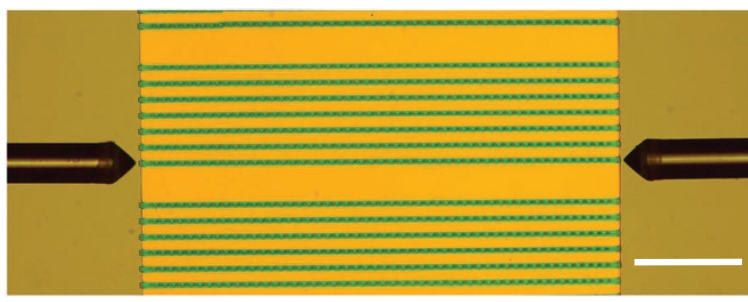

d

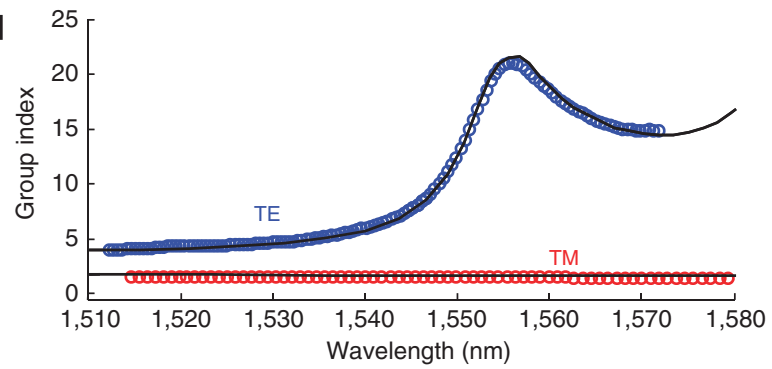

Figure 2 | PhC design. (a) SEM image of the tapered coupler at the end of the PhC waveguides (the scale bar is $1 \mu \mathrm{m}$ ). (b) Optical image of the chip containing the photonic crystal waveguides (green) and the coupling lensed fibres (the scale bar is $250 \mu \mathrm{m}$ ). (c) Design of the PhC waveguide with the anti-symmetric shift (of magnitude $T \times a$ ) of the first row of holes. (d) Measured (markers) and calculated (solid) dispersion (group index versus wavelength) for the TE and TM modes, showing good agreement. For details, see Methods.

group index. Two samples, fabricated within the same processing batch and only differing by the design, are compared. It is apparent that the design used for the delay line performs much better than the older design, represented with the red pattern.

Consistently with the discussion above, we have assessed the performance of the $\mathrm{PhC}$ waveguide as a delay-line for microwave signals by measuring both the RF power and delay as a function of the wavelength of the optical carrier for the TE polarization mode (see Methods). The baseline used for the normalization was obtained by measuring the transfer function of the microwave link when the $\mathrm{PhC}$ was by-passed. The delay, which is defined as $\tau_{\mathrm{RF}}=\mathrm{d} k / \mathrm{d} \omega$ and is plotted in Fig. $3 \mathrm{c}$, involves the measurement of the microwave signal phase. Consistent with the interferometric measurements in Fig. 3a, a delay increase of 70 ps was achieved by sweeping the carrier wavelength from 1,532 to $1,552 \mathrm{~nm}$. Besides, a flat response was obtained over the entire $(0-50 \mathrm{GHz})$ RF frequency band of the microwave link, whatever the delay. The microwave bandwidth was limited by the measurement equipment (the modulator, detector and vector network analyzer (VNA)). Thereby, no appreciable signal degradation was introduced by the $\mathrm{PhC}$ waveguide.

The amplitude transfer function is shown in Fig. 3d. A power variation of $7 \mathrm{~dB}$, corresponding to a $3.5-\mathrm{dB}$ change of the optical transmission, was experienced by the RF signal while tuning the delay by $70 \mathrm{ps}$, which is in perfect agreement with the optical transmission measurements described above. This figure guarantees a range of incremental delays between two different wavelengths, which is enough for filtering signals in the $10-100-\mathrm{GHz}$ band. From the application standpoint, this change in amplitude, which can be easily compensated, is relatively small compared with other photonic approaches, particularly those based on resonant-type schemes. This point has considerable practical implications. The large bandwidth handling capability is a clear advantage. This large electrical bandwidth is still a small fraction of the optical transmission band $(20 \mathrm{~nm}$, hence about $3 \mathrm{THz})$, which explains why distortion can be reduced to a manageable level.

Demonstration of filtering in the microwave domain. In our simplest implementation of the filter on a PhC chip, we consider a two-tap MWP notch filter exploiting the group index disparity between the TE and TM modes. The principle is illustrated in Fig. 4a. The key point is that the dispersion of the TM is negligible when compared with the TE mode. Therefore, when the optical carrier is coupled to the two polarization modes, the modulation envelope experiences a relative delay between the TE and TM taps, which is controlled by the carrier wavelength. In addition, by properly adjusting the polarization state of the input optical signal, the relative weight of the two taps is also controlled.

The generic layout of this polarization multiplexing PhC-based MWP filter is illustrated in Fig. 4b. The optical carrier, modulated by the microwave signal $s_{i}(t)$, is polarized to be coupled into both the TE and TM modes of the PhC waveguide. The output electrical signal $s_{o}(t)$, after propagation through the waveguide and photodetection, results from the incoherent superposition of the intensities of the TE and TM modes. The contribution carried by the TM mode experiences a group delay $\tau_{\mathrm{TM}}=L \times n_{\mathrm{g}, \mathrm{TM}} / \mathcal{c}$ ( $L$ is waveguide length), with $n_{\mathrm{g}, \mathrm{TM}}$ close to the effective index of the $\mathrm{PhC}$ slab, roughly $\sim 2$, weakly dependent on the wavelength of the optical carrier. Conversely, the signal carried by the TE mode is delayed by $\tau_{\mathrm{TE}}=L \times n_{\mathrm{g}, \mathrm{TE}}(\lambda) / c$, with $n_{\mathrm{g}, \mathrm{TE}}$ spanning between 5 and 20 (Fig. $2 \mathrm{~d}$ ).

The filtered electrical signal at the output of the photodiode is then:

$$
s_{o}(t)=s_{i}\left(t-\tau_{\mathrm{TM}}\right)+s_{i}\left(t-\tau_{\mathrm{TE}}\right),
$$

which corresponds, in the microwave frequency domain, to the filter transfer function $H(f)$ :

$$
H(f)=1+e^{-j\left(\frac{2 \pi f}{\mathrm{FSR}}\right)}
$$

being $f$ the microwave frequency and FSR the free spectral range of the filter, equal to $c /\left(L\left(n_{\mathrm{g}, \mathrm{TE}}(\lambda)-n_{\mathrm{g}, \mathrm{TM}}\right)\right)$. By combining these equations with the calculated and measured dispersion curves for the TE and TM modes in Fig. 2d, the expected response of the filter is readily obtained (see Fig. 4c).

Experimental results are in good agreement with the theory, particularly when the delay-line is operated with the group index below 

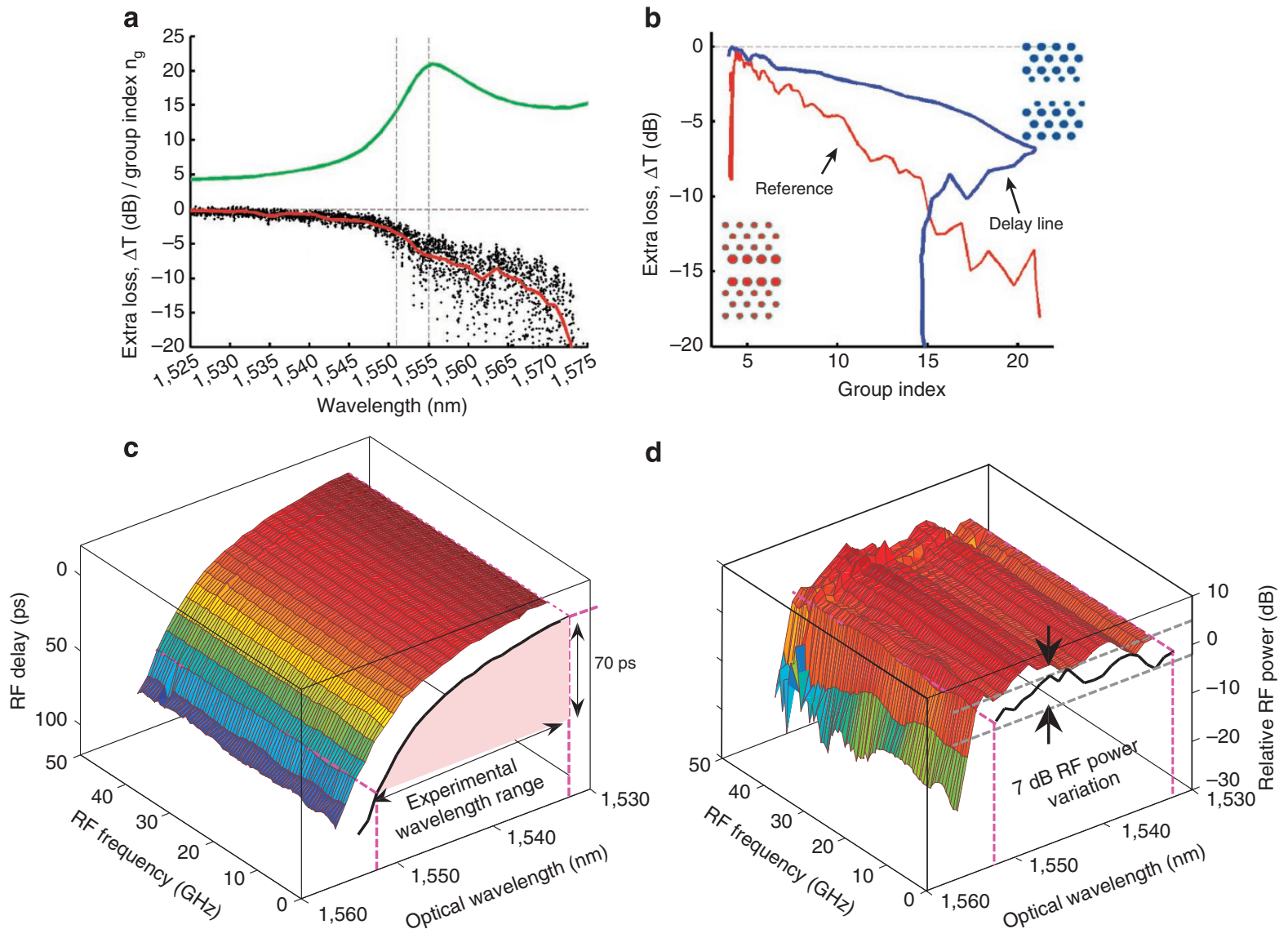

Figure 3 | Experimental characterization of the PhC waveguides. (a) Dispersion (group index versus wavelength, green solid line) and (red) excess transmission loss (that is, transmission normalized to maximum) of the TE even mode of the waveguide corresponding to the design with anti-symmetric shift (see Fig. 2c). The waveguide length is $1.5 \mathrm{~mm}$. The vertical dashed lines correspond to the +3.5 and +7 -dB extra-loss level and connect to the corresponding group index (14 and 21) and delay ( 70 and 105 ps). (b) Excess loss versus the group index for the waveguide used for the delay line (blue, same data as in Fig. 3a) compared with a reference sample with different design (red) used in the experiment by Roy ${ }^{35}$. (c,d) Measurements in the microwave domain of the sample referenced in Fig. 2d. Relative delay (c) and power variation (d) of the microwave signal as a function of the wavelength of the optical carrier and the microwave frequency.

a

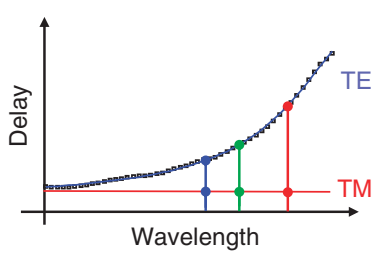

b

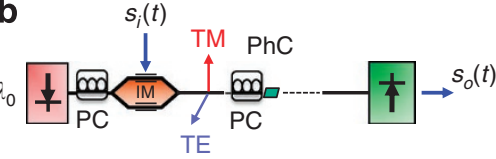

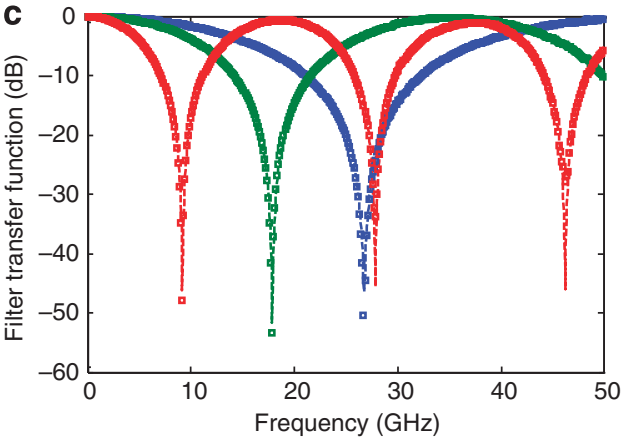

Figure 4 | Polarization-multiplex-based two-tap MWP filter. (a) The delay profiles, based on the experimental results obtained for the TE mode, for the TE and TM modes as a function of wavelength, for illustrative purposes of the filter operation. The TE and TM delays that correspond to the set of wavelengths selected for the filter experimental demonstration are indicated by colored dots, respectively, in blue, green and red for wavelengths of 1,544.5, 1,547 and 1,550.8 nm.

(b) Experimental setup. A TLS emitting at $\lambda_{0}$ is modulated via a Mach-Zehnder modulator $(I M)$ and carries the input electrical signal $s_{i}(t)$. A polarization controller is employed to optimize the power launched from the laser into the modulator. A second polarization controller ensures that both TE and TM polarizations are launched into the $\mathrm{PhC}$. A photodetector then provides the output filtered electrical signal $s_{0}(t)$. (c) Calculated (solid lines) and measured (squares) results for the filter response as a function of the wavelength. Blue, green and red markers, respectively, stand for 1,531, 1,537 and 1,542 nm optical wavelength.

10 (blue and green curves in Fig. 4c). Besides, it must be pointed out that, whatever the target frequency of the filter, a notch depth of $50 \mathrm{~dB}$ is achieved. This is a clear benefit of the integrated nature of the delay line. A small deviation from the theory is observed at larger frequencies, corresponding to a decrease of the transmission minimum to $30 \mathrm{~dB}$. This is related to the residual distortion of the 
a

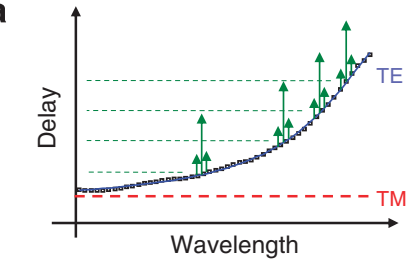

b

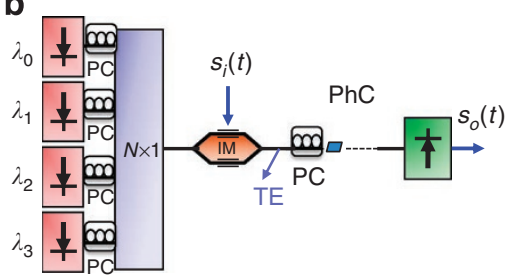

C

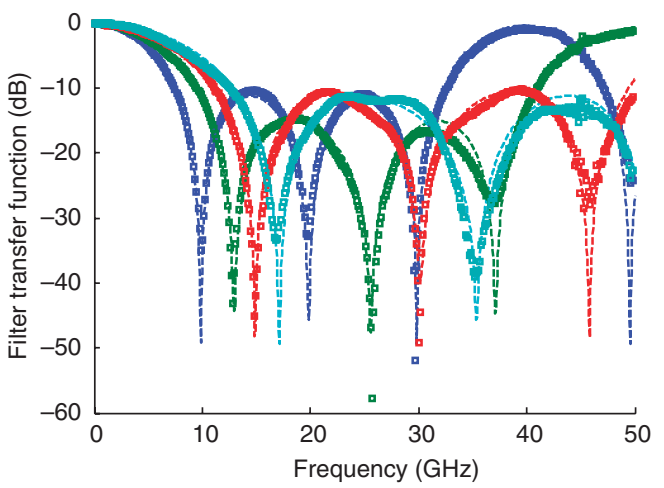

Figure 5 | Multi-wavelengh-based four-tap MWP filter. (a) The delay profiles, based on the experimental results obtained for the TE mode, for the TE and TM modes as a function of the wavelength for illustrative purposes of the filter operation. The green arrows show the spectral placement of the carriers and modulation sidebands (at $50 \mathrm{GHz}$ ) for all the four taps within the TE delay profile. This wavelength set is related to the green markers in figure (c), and corresponds to a constant group delay of $20 \mathrm{ps}$ from tap to tap, which means a free spectral range of $50 \mathrm{GHz}$ in the green filter transfer function in (c). (b) Experimental setup of the multi-wavelength filter. Four TLS ( $\lambda_{0}$ to $\left.\lambda_{3}\right)$ are combined and intensity modulated to carry the input electrical signal $s_{i}(t)$. The four wavelengths are then coupled into the photonic crystal delay line, along the TE mode, the RF delay for each tap being therefore a function of the tap wavelength. The polarization controllers perform the same functions as in the case of a single optical source. A photodetector provides the output filtered electrical signal $s_{0}(t)$. (c) Calculated (dashed lines) and measured (colored squares) results for the filter response with different spectral scenarios of the emission wavelengths. The experimental wavelengths sets are 1,530, 1,546.4, 1,550.4, 1,552.6 nm (blue), 1,541.5, 1,548.9, 1,551, 1,552.7 nm (green), $1,545.2,1,549.9,1,551.2,1,552.6 \mathrm{~nm}$ (red) and 1,546.8, 1,550.4, 1,551.5, 1,552.6 nm (cyan).

transfer function (Fig. 2d), which makes it difficult to adjust the tap weights perfectly all over the RF domain. More precisely; if $\alpha$ is the ratio of the electrical power between the taps, the filter extinction ratio $\mathrm{ER}$, or notch depth, is given by $\mathrm{ER}=(1+\alpha)^{2} /(1-\alpha)^{2}$. A $30-\mathrm{dB}$ notch depth thus corresponds to a $0.6-\mathrm{dB}$ RF power fluctuation in the RF frequency span, which is precisely what is measured from Fig. 2 d.

A more powerful configuration for band-pass filtering, in terms of the quality (Q)-factor and design flexibility, entails a larger number of taps. The straightforward implementation, which we have chosen here, consists of a single dispersive channel and multi-carriers optical source (for example, a comb or wavelength division multiplexing source), all modulated by the same microwave signal. This concept is illustrated in Fig. 5a and it was implemented using a long chirped $\mathrm{FBG}^{25}$ with maximum length about $40 \mathrm{~cm}$ (ref. 12). Equivalent configurations use dispersive optical fibre links (DOFL), with lengths up to $20 \mathrm{~km}$ (ref. 38), or dispersion-compensating fibres (DCFs) ${ }^{23}$.

The same concept is here implemented by replacing the FBG/ DOFL/DCF with an integrated PhC dispersive waveguide, thereby reducing the footprint of the key optical component by two orders of magnitude as compared with the FBG and six orders of magnitude as compared with the DOFL case. The layout of the four-tap PhCbased MWP filter (see Fig. 5b) consists of four tunable lasers followed by a common modulator. The four replicas of the microwave signal are coupled into the TE mode of the $\mathrm{PhC}$ waveguide and, upon propagation, experience different delays, set to form uniform time intervals $\Delta \tau$ by a suitable choice of the carrier wavelengths. As the carriers are not correlated together, the detected electrical signal is the incoherent sum of the optical intensities of the four paths and the corresponding transfer function in the microwave filter is:

$$
H(f)=\sum_{k=0}^{3} a_{k} \exp -j k\left(\frac{2 \pi f}{\mathrm{FSR}}\right.
$$

where FSR $=1 / \Delta \tau$, and $a_{k}$ are the tap weights, adjusted by controlling the relative intensities of the tunable lasers. In the experimental demonstration reported here, all the filter taps $a_{k}$ were set to the same power level (that is, uniformly apodized filter), but variation of coefficients, leading to transfer function reconfiguration, is straightforward ${ }^{5}$. Hence, with a single tunable delay line with a maximum delay $\tau_{\max }$ and $N$ taps, the FSR can be tuned from $(N-1) / \tau_{\max }$ to infinity. The experimental result is reported in Fig. $5 \mathrm{c}$ and it is in very good agreement with the model. As we limited the group delay to $80 \mathrm{ps}$, spectral range of the filter is adjusted from 40 to $70 \mathrm{GHz}$. The maximum extinction ratio is $50 \mathrm{~dB}$, whereas the MSSL ratio is about $10 \mathrm{~dB}$. This figure can be improved by increasing the number of channels; however, at the expense of the lower bound of the tuning range. The distortion of the RF signal is very low over a broad spectral range $(0-50 \mathrm{GHz})$.

Towards integration. With the demonstration of the suitability of a $\mathrm{PhC}$ waveguide as a delay line for microwave devices, all the key functionalities of the experimental setup described Fig. 5 could now be assembled in an integrated device. Indeed, optical modulators, photodetectors, splitters/combiners and laser sources are now available in integrated photonics platforms. In particular, integrated tunable laser sources (TLS) have recently been demonstrated on a silicon platform, through hybrid integration, which exhibit $45 \mathrm{~nm}$ tuning range around $1.5 \mu \mathrm{m}$. Alternatively, a similar tunability has been achieved on an InP platform with $13 \mathrm{dBm}$ output power when followed by a co-integrated semiconductor optical amplifier $(\mathrm{SOA})^{39}$. High-speed intensity modulation on a silicon platform has also been extensively studied in the past years and is still a very active topic. Modulation of $40 \mathrm{~Gb} \mathrm{~s}^{-1}$ has recently been achieved within the Helios project consortium with a Mach-Zehnder type device ${ }^{40}$. The electro-absorption modulators in germanium PIN on SOI (silicon on insulator) also exhibit interesting features of high bandwidth (up to $30 \mathrm{GHz}$ ), small footprint (a few tens of microns in length), low insertion loss (less than $5 \mathrm{~dB}$ ) and extinction ratio up to $7 \mathrm{~dB}$ (ref. 41). Fast (bandwidth $>40 \mathrm{~Gb} \mathrm{~s}^{-1}$ ) and efficient (responsivity $=0.8 \mathrm{~A} \mathrm{~W}^{-1}$ ) photodetectors have also been demonstrated on the same platform (germanium on $\mathrm{SOI})^{42}$. 
Thus, the availability of a low-loss and tunable delay line, providing a large RF bandwidth, as demonstrated here, would complete the toolbox for the full integration of a MWPs filter. A possible layout representing the integrated counterpart of the experiment described above is shown in Fig. 6.

Beyond the issue of manufacturability, the prospective integrated device must be analyzed with respect to the expected performances, in particular the power budget of the entire microwave link. In a photonic microwave device, this scales as the square of the total optical losses and as the square of the efficiency of the electro-optic intensity modulator. According to the literature, a 4:1 power combiner (for example, multimode interference coupler) and the intensity modulation (including insertion losses and bias point losses) exhibit cumulated optical losses in the range of $8 \mathrm{~dB}^{43,44}$. Including the $\mathrm{PhC}$ delay line, one ends up with $\sim 15 \mathrm{~dB}$ of total optical losses (a more accurate estimate must take into account the on-chip coupling loss, likely smaller than fibre-PhC insertion losses).

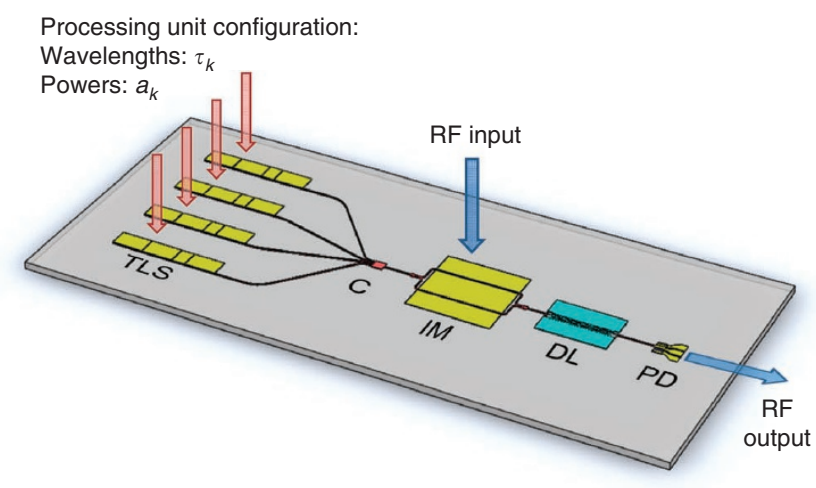

Figure 6 | Layout of a fully integrated microwave photonics signal processor. The weighting coefficients $a_{k}$ of the impulse response of the processing unit are programmed through the relative power levels of the TLSs, whereas the selected wavelengths set the taps group delays $\tau_{k}$, and hence the filter FSR. DL, photonic crystal delay line; IM, intensity modulator; PD, photodiode; Semiconductor optical amplifiers C, 4:1 optical combiner; TLS, tunable laser source.
Thus, considering an optical power of $10 \mathrm{dBm}$ at the output of each of the TLS, the RF power budget of the device (that is, the ratio between output and input RF power) is approximately $-45 \mathrm{~dB}$. This value is compatible with most practical system implementations. As a comparison, the power budget of a basic MWPs link (laser modulator fibre detector using discrete components) with $10 \mathrm{dBm}$ optical power is already in the range of $-25 \mathrm{~dB}$.

\section{Discussion}

The PhCs-based filter is compared to other competing MWP implementations, as well as against purely electronic microwave devices. A summary of relevant results can be found in Table 1. First, a single $\mathrm{PhC}$ waveguide enables a very large operating bandwidth, mainly limited by the disorder-induced distortion of the transmission, the bandwidth of the photodetectors and group velocity dispersion. The $50-\mathrm{GHz}$ bandwidth reported here could be improved, through single-sideband modulation for instance. Although filters based on stimulated Brillouin scattering can achieve similar bandwidths, the integration is not possible and the power consumption (inherent to the stimulated Brillouin scattering effect) is large ${ }^{45,46}$. Power consumption is also a concern for filters based on SOAs ${ }^{47}$.

Because the proposed filter is based on a dispersive delay line, its performance can be affected by the power fading effect if doublesideband modulation is employed ${ }^{1-3}$. A straightforward measure to eliminate this limitation is to use single-sideband modulation ${ }^{1-3}$, which adds no significant complexity to the modulator. However, even if double sideband modulation is employed, the fact that the delay line length $L$ is very small and that the power fading effect penalty is more severe the higher the product of the dispersion $D$ and $L$ is makes it possible to observe no significant penalty within the spectral range of interest. In our particular case, the power fading penalty was significant only above $80 \mathrm{GHz}$, which was outside our measurement range.

The hybrid integrated approach ${ }^{48}$ provides photodetectorlimited bandwidth, combined with small footprint and very low loss. In addition, the tunability speed is very high. However, the tuning range is limited and the power consumption is not negligible as the structure integrates several active elements, such as SOAs. In terms of rejection level and bandpass/rejection bandwidth, the results reported here are still behind some of those achieved by

Table 1 | Comparison of the microwave filter with other technologies against the figures of merit.

\begin{tabular}{|c|c|c|c|c|c|c|c|c|c|c|}
\hline Technology & Filter type & $\begin{array}{l}\text { Bandpass/ } \\
\text { rejected } \\
\text { bandwidth } \\
(\mathrm{GHz})\end{array}$ & $\begin{array}{l}\text { Operational } \\
\text { bandwidth } \\
(\mathrm{GHz})\end{array}$ & $\begin{array}{l}\text { Central } \\
\text { frequency } \\
\text { (GHz) }\end{array}$ & Size & $\begin{array}{l}\text { Insertion } \\
\text { loss (dB) }\end{array}$ & $\begin{array}{l}\text { Rejection } \\
\text { level (dB) }\end{array}$ & $\begin{array}{l}\text { Tunability } \\
\text { speed }\end{array}$ & $\begin{array}{l}\text { Tunability } \\
\text { range (\%) }\end{array}$ & $\begin{array}{c}\text { Power } \\
\text { consumption }\end{array}$ \\
\hline $\mathrm{PhC}$ & $\begin{array}{l}\text { Bandpass/ } \\
\text { Notch }\end{array}$ & $8 / 1-2$ & $\begin{array}{c}>40 / P D \\
\text { and GVD } \\
\text { limited }\end{array}$ & $\begin{array}{c}>40 / P D \\
\text { and GVD } \\
\text { limited }\end{array}$ & $\sim \mathrm{mm}$ & 9 & $>10 / 50$ & $\mathrm{~ms}$ & 100 & - \\
\hline $\mathrm{SOA}^{47}$ & Notch & 0.001 & 0.03 & 30 & $\sim \mathrm{mm}$ & 10 & $>30$ & ps & 100 & Medium \\
\hline Brillouin 45 & Notch & $\sim 0.1$ & PD limited & PD limited & $\sim$ km fibre & 0.5 & $>30$ & $\mu \mathrm{s}$ & 100 & High \\
\hline $\mathrm{FBG}^{51}$ & Bandpass & 0.5 & 20 & $6-7.5$ & $\sim \mathrm{cm}$ & 6 & $>10$ & $\mathrm{~ms}$ & 70 & - \\
\hline Brillouin 46 & Bandpass & 0.02 & PD limited & PD limited & $\sim$ km fibre & 0 & $>30$ & $\mu \mathrm{s}$ & 100 & High \\
\hline $\mathrm{LCoS}^{52}$ & Bandpass & 0.3 & $<20$ & $2-3$ & $\sim \mathrm{cm}$ & 1.75 & $>30$ & s & 25 & - \\
\hline $\begin{array}{l}\text { Frequency } \\
\text { comb }^{23}\end{array}$ & Bandpass & $0.17-0.36$ & $\begin{array}{l}10 \text { (RF gen. } \\
\text { limited) }\end{array}$ & 5 & $\sim$ km fibre & 1 & $>60$ & ns & 100 & - \\
\hline SOI ring 20 & Notch & 0.01 & 1 & 20 & $\sim 0.2 \mathrm{~mm}^{2}$ & 10 & $>30$ & $\mathrm{~ms}$ & 77 & - \\
\hline SOl ring 53 & Notch & 0.01 & 1 & 1.7 & $\sim 30 \mathrm{~mm}^{2}$ & 10 & $>25$ & $\mathrm{~ms}$ & 100 & - \\
\hline Hybrid $^{42}$ & Bandpass & $3-14$ & PD limited & $9-37$ & few $\mathrm{mm}^{2}$ & 0 & $>25$ & ps & 58 & Medium \\
\hline MEMS $^{8}$ & Bandpass & 0.06 & 0.6 & $1.2-1.6$ & $\sim 20 \mathrm{~cm}^{2}$ & 4.1 & $>15$ & $\mathrm{~ms}$ & 28 & Low \\
\hline $\mathrm{MW}^{\star}$ & Bandpass & 0.02 & 0.4 & $1.8-2.2$ & $\sim \mathrm{cm}^{3}$ & 2 & $>30$ & $\mathrm{~s}$ & 20 & - \\
\hline$M W_{\dagger}$ & Notch & 0.015 & 1 & $3-4$ & $\sim \mathrm{cm}^{3}$ & 0.5 & $>40$ & $\mathrm{~s}$ & 29 & - \\
\hline
\end{tabular}

*Data corresponds to a microwave tunable bandpass filter manufactured by Lorch operating in the $24-3,000-\mathrm{MHz}$ range.

$\dagger$ Data corresponds to a microwave tunable notch filer manufactured by KL Microwave operating in the 3,000-4,000- $\mathrm{MHz}$ range. 
using discrete component technologies. However, there is no fundamental limit to the improvement of these figures, rather, as the discrete filter is inherently scalable, the compactness of PhCs is an obvious advantage.

In this implementation, the tuning speed depends on the optical source, which ultimately is limited by the carrier injection dynamics, namely in the nanosecond time scale. As $200 \mathrm{~ns}$ thermooptical switching was demonstrated in $\mathrm{PhC}$ waveguides ${ }^{49}$, it is expected that our filter could be operated with a fixed wavelength source and tuned within comparable time scales.

The bandpass width of our four-tap PhC-based filter is $8 \mathrm{GHz}$, which is adequate in the context of broadband signals processing, but could be improved depending on the number of taps. We point out that Supradeepa et al. ${ }^{23}$ demonstrated a MWP filter based on a tailored optical comb source, combined with a DCF, with remarkable fast and broad tuning, high degree of reconfigurability and selectivity, owing to the large number of optical carriers used. The implementation of this design in our device is straightforward and would greatly benefit from the small footprint of the $\mathrm{PhC}$ delay line. A fully integrated device could be feasible. However, a linear dispersion profile is required as the frequency combs are equally spaced in frequency. In our device, the dispersion is nearly linear between 1,548 and $1,553 \mathrm{~nm}$, with an average group velocity dispersion of $\sim 10 \mathrm{ps} \mathrm{nm}^{-1}$. If the frequency comb is spaced by $0.4 \mathrm{~nm}(\sim 50 \mathrm{GHz})$, 12 optical carriers could be accommodated, resulting into a delay interval $\tau /(N-1) \approx 4 \mathrm{ps}$, that is, the FSR is $250 \mathrm{GHz}$. A more appropriate design of the $\mathrm{PhC}$ delay line would improve this figure.

\section{Methods}

Description of the figures of merit. In bandpass filters, bandwidth is defined as the $-3-\mathrm{dB}$ or the full width half maximum of the passband, whereas for notch filters it refers to the spectral width at the rejection level of $20 \mathrm{~dB}$ with respect to the maximum. The operational bandwidth is defined as the spectral range within which the filter response can be tuned (it may not necessarily be equal to the spectral period or FSR). The central frequency refers, in the case of bandpass filters, to the position of the first maxima without considering baseband, whereas in notch-type filters, it corresponds to the frequency where the operational bandwidth centres. Size and insertion loss address here only the subsystem that enables filter tunability, that is, the delay line in our PhC-based filter implementation. In addition, insertion loss refers to the overall optical loss in MWP and electrical loss in MW filters. The rejection level indicates the MSSL ratio for bandpass filters, while it addresses the transmission minimum in the notch-type filters. The tunability range accounts for the FSR in both bandpass and notch-type filters. The lowest timing scale to perform filter tunability is given by the tunability speed. The power consumption only refers to the power required to operate the delay line. Here, 'high' indicates more than $100 \mathrm{~mW}$, whereas 'medium' indicates a power consumption range in between 10 and $100 \mathrm{~mW}$.

PhC waveguide fabrication. $\mathrm{PhC}$ waveguides were fabricated using a planar process. First, a 200-nm-thick mask of silica was deposited on a semiconductor structure containing a 180 -nm-thick layer of InGaP grown by metal-organic chemical vapour deposition on GaAs. A poly(methyl methacrylate) (PMMA) resist was spun on it and exposed using e-beam writer. After etching of the silica mask, the III-V material was processed via inductively coupled plasma etching and finally a self-standing membrane was obtained by wet etching of the underlying layer of GaAs. The waveguide design is shown in Fig. 1c. The lattice period $a$ is $482 \mathrm{~nm}$, whereas the radius of the holes is $0.26 a$. The first row of holes exhibits a slightly smaller radius $(0.25 a)$ and is shifted on opposite directions along the waveguide axis by $0.15 a$.

Calculation of the PhC dispersion. We used our own implementation of the 3D finite differences in time domain method with periodic boundary conditions; with $x$ the propagation direction of the Bloch mode and $a$ the period of the $\mathrm{PhC}$ lattice. The size of the unit cell for the calculation was: $a \times 20 a \times 6 a$ and the mesh resolution was down to $a / 20$. The estimated relative accuracy in the frequency was better than $1 / 1000$.

PhC characterization. The dispersion of the waveguide was measured using a TLS, which was swept over its entire spectral range $(1,510-1,630 \mathrm{~nm})$. The signal was then split to build up a Mach-Zehnder interferometer (MZI), with one of the arms containing the $\mathrm{PhC}$ waveguide. The two outputs of the MZI were fed to a balanced photodetectors (characterized by a trans-impedance gain of $10^{5}$ ) and the differential signal was digitized with a 14-bit analog-to-digital converter. The wavelength was monitored with a reference interferometer. The resulting interferogram $S(f)$, with $f$ the optical frequency, was filtered using a Gaussian window centred at $f_{j}$ and Fourier-transformed to obtain the time-resolved transmission $S_{j}(t)$. The group delay $t\left(f_{j}\right)$ is defined as the peak of $S_{j}(t)$.

MWP link characterization. The delay introduced by the $\mathrm{PhC}$ waveguide and the corresponding loss were measured in the RF domain by inserting the device into a MWP link. The optical carrier of the MWP link was generated by a TLS. The RF signal was fed into the link through the RF input of a $50-\mathrm{GHz}$ bandwidth Mach-Zehnder electro-optic modulator (MZM), which modulates the optical carrier intensity. The laser delivered $5 \mathrm{dBm}$ optical power to the input of the MZM electro-optic modulator. Insertion losses of the MZM where $5 \mathrm{~dB}$ resulting in a $0-\mathrm{dBm}$ input power to the polarization controller deployed to adjust the polarization state at the input of the $\mathrm{PhC}$ waveguide. Lensed fibres were used for input and output optical coupling. A high-speed photodetector was followed by an RF amplifier to compensate for the loss introduced by the MZM and the PhC device.

The resulting MWP link was characterized as a two-port electrical component using a VNA whose output and input ports were, respectively, connected to the MZM RF input and the RF amplifier output. The S21 parameter measured by the VNA provided both the RF power and phase transfer function from $10 \mathrm{MHz}$ to $50 \mathrm{GHz}$. The measurements given in Fig. $2 \mathrm{c}, \mathrm{d}$ are referenced to a first acquisition performed at the lowest optical wavelength, corresponding to a fast propagation regime in the $\mathrm{PhC}$ waveguide.

MWP filters characterization. The results for the two-tap filter implementation shown in Fig. 4 were measured by deploying the same experimental setup used for the MWP link characterization. On the other hand, the output signal of four tunable lasers was optically coupled prior the MZM for obtaining the four-tap filter response illustrated in Fig. 5. As the detuning is large with respect to the phase matching condition ${ }^{50}$, no significant nonlinear effects were observed in the $\mathrm{PhC}$ waveguide.

\section{References}

1. Seeds, A. Microwave photonics. IEEE Trans. Microwave Theory Tech. 50, 877-887 (2002).

2. Capmany, J. \& Novak, D. Microwave photonics combines two worlds. Nat. Photon 1, 319-330 (2007).

3. Yao, J. P. Microwave photonics. J. Lightwave Technol. 27, 314-335 (2009).

4. See special technology focus on microwave photonics. Nat. Photon 5, 723-736 (2011).

5. Capmany, J., Ortega, B. \& Pastor, D. A tutorial on microwave photonic filters J. Lightwave. Technol. 24, 201-229 (2006).

6. Long, J. et al. A tunable microstrip bandpass filter with two independently adjustable transmission zeros. IEEE Microw. Wireless Compon. Lett. 21, 74-76 (2011).

7. Velez, A. et al. Tunable coplanar waveguide band-stop and band-pass filters based on open split ring resonators and open complementary split ring resonators. IEEE Microw. Antennas Propag. 5, 277-281 (2011).

8. Sekar, V., Armendariz, M. \& Entesari, K. A 1.2-1.6-GHz substrate-integrated waveguide RF MEMS tunable filter. IEEE Trans. Microwave Theory Tech. 59, 866-876 (2011)

9. Rafique, M. R. et al. Miniaturized superconducting microwave filters. Supercond. Sci. Technol. 21, 075004 (2008).

10. Velu, G. et al. A $360^{\circ}$ BST phase shifter with moderate bias voltage at $30 \mathrm{GHz}$ IEEE Trans. Microwave Theory Tech. 55, 438-444 (2007)

11. Koh, K. J. \& Rebeiz, G. M. A 6-18 GHz active phase shifter. In Proceedings IEEE Microwave Symposium Digest 792-795 (2010).

12. Capmany, J., Pastor, D. \& Ortega, B. New and flexible fiber-optic delay-line filters using chirped Bragg gratings and laser arrays. IEEE Trans. Microwave Theory Tech. 47, 1321-1326 (1999).

13. Minasian, R. A. Photonic signal processing of microwave signals. IEEE Trans Microwave Theory Tech. 54, 832-846 (2006).

14. Dai, Y. \& Yao, J. P. Nonuniformly-spaced photonic microwave delay-line filter. Opt. Express 16, 4713-4718 (2008).

15. Hamidi, E., Leaird, D. E. \& Weiner, A. M. Tunable programmable microwave photonic filters based on an optical frequency comb. IEEE Trans. Microwave Theory Tech. 58, 3269-3278 (2010).

16. Chan, E. H. W. \& Minasian, R. A. Coherence-free high-resolution RF/ microwave photonic bandpass filter with high skirt selectivity and high stopband attenuation. J. Lightwave Technol. 28, 1646-1651 (2010).

17. Norberg, E. J. et al. Programmable photonic microwave filters monolithically integrated in InPinGaAsP. J. Lightwave. Technol. 29, 1611-1619 (2011).

18. Chen, H. W. et al. Integrated microwave photonic filter on a hybrid silicon platform. IEEE Trans. Microwave Theory Tech. 58, 3213-3219 (2010).

19. Dong, P. et al. GHz-bandwidth optical filters based on high-order silicon ring resonators. Opt. Express 18, 23784-23789 (2010).

20. Lloret, J. et al. Tunable complex-valued multi-tap microwave photonic filter based on single silicon-on-insulator microring resonator. Opt. Express 19, 12402-12407 (2011). 
21. Notomi, M. et al. Extremely large group-velocity dispersion of line-defect waveguides in photonic crystal slabs. Phys. Rev. Lett. 87, 253902 (2001).

22. Knight, J. C. Photonic crystal fibres. Nature 424, 847-851 (2003).

23. Supradeepa, V. R. et al. Comb-based radiofrequency photonic filters with rapid tunability and high selectivity. Nat. Photon. 6, 186-194 (2012).

24. Capmany, J., Ortega, B., Pastor, D. \& Sales, S. Discrete-time optical processing of microwave signals. J. Lightwave Technol. 23, 702-723 (2005).

25. Hunter, D. B. \& Minasian, R. A Tunable microwave fiber-optic bandpass filters IEEE Photon. Tech. Lett. 11, 874-876 (1999).

26. Baba, T. Slow light in photonic crystals. Nat. Photon. 2, 465-473 (2008).

27. Kuramochi, E. et al. Disorder-induced scattering loss of line-defect waveguides in photonic crystal slabs. Phys. Rev B 72, 161318 (2005).

28. Ishikura, N., Baba, T., Kuramochi, E. \& Notomi, M. Large tunable fractional delay of slow light pulse and its application to fast optical correlator. Opt. Express 19, 24102-24108 (2011).

29. O'Faolain, L. et al. Loss engineered slow light waveguides. Opt. Express 18, 27627-27638 (2010)

30. Baron, A., Mazoyer, S., Smigaj, W. \& Lalanne, P. Attenuation Coefficient of Single-Mode Periodic Waveguides. Phys. Rev. Lett. 107, 153901 (2011)

31. Patterson, M. et al. Disorder-Induced Coherent Scattering in Slow-Light Photonic Crystal Waveguides. Phys. Rev. Lett. 102, 253903 (2009).

32. Mazoyer, S., Hugonin, J. P. \& Lalanne, P. Disorder-Induced Multiple Scattering in Photonic-Crystal Waveguides. Phys. Rev. Lett. 103, 063903 (2009).

33. Combrié, S. et al. Time-delay measurement in singlemode, low-loss photonic crystal waveguides. Electron. Lett. 42, 86-87 (2006).

34. Liang, J. et al. Wideband ultraflat slow light with large group index in a W1 photonic crystal waveguide. J. App. Phys. 110, 063103 (2011).

35 . Roy, S. Modeling the dispersion of the nonlinearity in slow mode photonic crystal waveguides. IEEE Photonics. Journal 4, 224-233 (2012).

36. Colman, P., Combrié, S. \& De Rossi, A. Control of dispersion in photonic crystal waveguides using group symmetry theory. Opt. Express 20, 13108-13114 (2012).

37. Vy Tran, Q., Combrié, S., Colman, P. \& De Rossi, A. Photonic crystal membrane waveguides with low insertion losses. Appl. Phys. Lett. 95, 061105 (2009)

38. Bolea, M., Mora, J., Ortega, B. \& Capmany, J. Highly chirped single-bandpass microwave photonic filter with reconfiguration capabilities. Opt. Express 19, 4566-4576 (2011).

39. Binetti, P. et al. Indium phosphide integrated circuits for coherent optical links. IEEE J. Quantum Electron. 48, 279-291 (2012).

40. Thomson, D. J. et al. High contrast $40 \mathrm{Gbit} / \mathrm{s}$ optical modulation in silicon. Opt. Express 19, 11507-11516 (2011).

41. Asghari, M. \& Krishnamoorthy, A. V. Energy efficient communication. Nat. Photon. 5, 268-270 (2011).

42. Vivien, L. et al. Zero-bias 40Gbit/s germanium waveguide photodetector on silicon. Opt. Express 20, 1096-1101 (2012).

43. Feng, N. N. et al. $30 \mathrm{GHz}$ Ge electro-absorption modulator integrated with $3 \mu \mathrm{m}$ silicon-on-insulator waveguide. Opt. Express 19, 7062-7067 (2011).

44. Trinh, P. D., Yegnanarayanan, S., Coppinger, F. \& Jalali, B. Compact multimode interference couplers in Silicon-on-insulator technology. Conference on Lasers and Electro-Optics CLEO '97CThV4, 441 (Baltimore, USA, 1997).
45. Loayssa, A., Capmany, J., Sagues, M. \& Mora, J. Demonstration of incoherent microwave photonic filters with all-optical complex coefficients. IEEE Photon. Tech. Lett. 18, 1744-1746 (2006).

46. Zhang, W. \& Minasian, R. A. Widely tunable single-passband microwave photonic filter based on stimulated Brillouin scattering. IEEE Photon. Tech. Lett. 23, 1775-1777 (2011).

47. Xue, W., Sales, S., Mork, J. \& Capmany, J. Widely tunable microwave photonic notch filter based on slow and fast light effects. IEEE Photon. Tech. Lett. 21, 167-169 (2009).

48. Norberg, E. J. et al. A monolithic programmable optical filter for RF signal processing. in Proceedings Microwave Photonics Conf. (Montreal, Canada, 2010).

49. Vlasov, Y. A., O’Boyle, M., Hamann, H. F. \& McNab, S. J. Active control of slow light on a chip with photonic crystal waveguides. Nature 438, 65-69 (2005).

50. Eckhouse, V. et al. Highly efficient four wave mixing in GaInP photonic crystal waveguides. Opt. Lett. 35, 1440-1442 (2010).

51. Sagues, M. et al. Multi-tap complex-coefficient incoherent microwave photonic filters based on optical single-sideband modulation and narrow band optical filtering. Opt. Express 16, 295-303 (2008).

52. Huang, T. X. H., Yi, X. \& Minasian, R. A. Single passband microwave photonic filter using continuous-time impulse response. Opt. Express 19, 6231-6242 (2011).

53. Burla, M. et al. On-chip CMOS compatible reconfigurable optical delay line with separate carrier tuning for microwave photonic signal processing. Opt. Express 19, 21475-21484 (2011).

\section{Acknowledgements}

This work was funded by the European Union under the project GOSPEL (grant 219299) and by the Valencian Government (Prometeo GVA 2008-92). We thank S. Hughes and P. Lalanne for enlightening discussion about the impact of disorder in photonic crystal waveguides.

\section{Author contributions}

J. Bourderionnet, Juan Sancho and Juan Lloret performed the experiment in the microwave domain and contributed to writing the paper; Salvador Sales, Ivana Gasulla and José Capmany contributed to writing the paper; S. Combrié and A. De Rossi optimized the design, performed the optical characterization of the PhC delay line and contributed to writing the paper; G. Lehoucq and S. Xavier fabricated the sample; P. Colman contributed to the design of the sample; D. Dolfi contributed to writing the paper; José Capmany, J. Bourderionnet, S. Combrié and A. De Rossi directed the project.

\section{Additional information}

Competing financial interests: The authors declare no competing financial interests.

Reprints and permission information is available online at http://npg.nature.com/ reprintsandpermissions/

How to cite this article: Sancho, J. et al. Integrable microwave filter based on a photonic crystal delay line. Nat. Commun. 3:1075 doi: 10.1038/ncomms2092 (2012). 